\section{Reports of Societies}

\section{HYPERTENSION}

At a joint meeting of the Liverpool Medical Institution and the Manchester Medical Society held in Manchester on March 5, with Prof. D. Dougal in the chair, there was a discussion on hypertension.

Dr. E. T. BAKER-BATES said that despite the fact that hypertension had been recognized for a century our knowledge of this leading cause of death was far from satisfactory. Hypertension could be produced experimentally by procedures that induced renal ischaemia. The significant conclusion was that hypertension could per se produce those vascular changes in the kidney and other organs formerly believed to be of inflammatory origin (Bright's disease). A vicious circle thus became established. On rare occasions this hypothesis had been confirmed in man by observing the relief of hypertension following removal of a diseased kidney. It now appeared that hypertension resulted from sustained arteriolar constriction brought about by a chemical agent (renin) liberated from ischaemic renal tissue.

The clinician must always satisfy himself that the patient's symptoms were due to a raised blood pressure. Neglect of this principle had caused much unnecessary invalidism. Treatment remained unsatisfactory. In the majority of cases guidance of the patient to ensure a reasonable attitude towards the condition was all that was possible or necessary. Excessive zeal was undesirable and often induced an anxiety state.

In recent studies, carried out independently in America and England, 33 preparations reputed to be capable of lowering the blood pressure did not produce significant reduction in a single case. Efforts should be made to keep the blood pressure as low as possible by restriction of activities, without making the patient an invalid. Sedatives, such as bromides or barbiturates, were indicated when there were nervousness, headaches, worry, or restlessness, but treatment by prolonged rest in bed, purgation, venesection, diet, baths, and diathermy was of negligible value. An artificial reduction in the blood pressure often made the patient feel worse. Finally, in no other chronic and progressive malady were common sense and insight into the weaknesses and fears of human nature more essential.

\section{Limitations of Surgery}

Mr. R. EDWARDS considered that, since the fundamental cause of essential hypertension was still quite unknown, any direct surgical attack upon the disease was not practicable at the moment, except in two instances: paroxysmal hypertension due to an adrenaline-secreting tumour, and rare cases of unilateral renal disease. There were two factors maintaining the hypertensive state: spasm in the first place and later sclerosis. If spasm could be controlled the sclerosis might be delayed: Vascular spasm could be relieved by division of the sympathetic nervous system. Division of the splanchnic nerves produced an extensive area of vasodilatation. This area could be increased by division also of the lower part of the dorsal sympathetic chain and the upper part of the lumbar sympathetic chain. This was the method Smithwick suggested. Adson, Craig, and Peet were content with a more limited operation.

The degree of spasm was best studied by the administration of thiopentone until a deep narcosis was produced, the blood pressure being recorded as each $1 \mathrm{ml}$, was administered. Full renal investigation was important. If considerable renal dysfunction was found it was unlikely that sympathectomy would benefit the patient, and sympathectomy was also of little value if cerebral damage was present. A history of coronary occlusion or anginal attacks did not contraindicate operation. Sympathectomy should not be performed in patients with a systolic pressure under $200 \mathrm{~mm}$. or a diastolic pressure of under $100 \mathrm{~mm}$. $\mathrm{Hg}$; but this opinion might be changed in view of the considerable success obtained in America with patients showing a lower blood pressure.

The operation was done on each side with an interval of two to three weeks between the operations. The anaesthetic used was of little moment so long as controlled respiration was main- tained, since the pleura might be entered at any time. The first 10 of his own 35 cases were treated by subdiaphragmatic splanchnicectomy, but severe and intractable post-operative vomiting led to its discontinuance. These were cases of advanced malignant hypertension in young people and the operations were performed some four years ago; all these patients were probably dead.

In the remaining 25 operations he resected the tenth and eleventh ribs posteriorly and removed a long section of the great splanchnic nerve and the sympathetic chain from the seventh dorsal ganglion down to the twelfth. An anterior transpleural approach suggested by Prof. Boyd had been utilized lately and this was an improvement on the posterior method. Delayed results were obtained in 16 cases; the remainder were too recent for assessment. There was a good immediate fall in blood pressure with a gradual rise in the succeeding months, but usually not to the original level. In a small number of cases the blood pressure had remained well down. In nearly all cases headache had been permanently relieved. The operation was not a cure for hypertension but was of considerable therapeutic value in selected cases.

\section{Familial Factors}

Prof. Robert Platt disagreed with some of the points made by the previous speakers. In essential hypertension psychological causes and food and drink played no part in aetiology. So-called menopausal hypertension was not due to the menopause but was discovered at that time because menopausal symptoms led to the blood pressure being taken. In these cases the hypertension did not diminish later on. The most important factor in essential hypertension was familial. More than $90 \%$ of cases in which the data were obtainable had a history of hypertension in one or both parents-it was a Mendelian dominant. In secondary hypertension the familial pattern was entirely different.

He considered that malignant and benign hypertension were the same disease and had the same hereditary background. Cases of so-called malignant hypertension in young persons were nearly always secondary to urological disease. In assessing the value of surgical treatment he said that unless the diastolic pressure was reduced to below $110 \mathrm{~mm}$. the prognosis was probably unaltered. The headaches could be relieved by medical treatment.

Prof. Geoffrey JefFerson said that in his patients the blood pressure did not fall very much afterwards (the diastolic pressure very rarely going below $110 \mathrm{~mm}$.) and in nearly all cases the blood pressure rose again later. Of 12 of his recent cases only two did reasonably -well. One, a child whose blood pressure fell from $180 / 120 \mathrm{~mm}$. to $120 / 80 \mathrm{~mm}$. after two years, was still very unwell. The second was a young woman whose vision returned, with a blood pressure of $155 / 110 \mathrm{~mm}$., two years later. He doubted whether these poor results justified the extensive surgical procedure necessary.

Prof. A. M. BoyD referred to his experience with Adson's operation. This gave some symptomatic relief but there was persistence of the hyperpiesis after six months. He had been using the transpleural approach, the double operation being very extensive. He thought that cyclopropane was the best anaesthetic to use, and said that after this operation it was necessary to remove the pleural effusions once or twice.

Prof. C. Wells referred to his few cases done recently. He felt that sympathectomy was not the complete answer since it did not cure hypertension, but he thought that it might relieve the patient of some of his symptoms and so make life more bearable for a few months.

The Danish Medical Society has organized a comprehensive scheme of research with regard to penicillin, and in Nordisk Medicin for March 21 many pages (716-738) are devoted to some of the most recent Danish experiences in this field. Dr. N. B. Krarup points out here that continuous intravenous and intramuscular injections of penicillin have not found great favour in Denmark, where the procedure usually preferred is the intramuscular injection every third hour. The treatment of 21 cases of diphtheria with penicillin was not very encouraging, but the results in cases of scarlet fever at the Blegdam Fever Hospital have been remarkably good. 